\title{
Uso de las Redes Neuronales Artificiales en el Modelado del Ensayo de Resistencia a Compresión de Concreto de Construcción según la Norma ASTM C39/C 39M
}

\author{
Luis Acuña(1), Ana V. Torre ${ }^{(2)}$, Isabel Moromi ${ }^{(2)}$ y Francisco García(3) \\ (1) Facultad de Ingeniería Industrial y de Sistemas, Universidad Nacional de Ingeniería, \\ Av. Túpac Amaru, 210. Lima 25-Perú (e-mail: lacuna@uni.edu.pe) \\ (2) Facultad de Ingeniería Civil, Universidad Nacional de Ingeniería, Av. Túpac Amaru, 210, \\ Lima 25-Perú (e-mail: anatorre@uni.edu.pe, imoromi@yahoo.com) \\ (3) Depto. Ingeniería Forestal, Universidad Politécnica de Madrid, Ciudad Universitaria s/n, \\ 28040 Madrid-España (e-mail: francisco.garcia@upm.es)
}

Recibido Dic. 16, 2013; Aceptado Ene. 15, 2014; Versión final recibida Mar. 3, 2014

\section{Resumen}

Se proponen dos modelos de redes neuronales artificiales para la predicción del resultado del ensayo a compresión de un concreto de construcción tras el periodo de curado a partir de datos de fabricación fácilmente medibles. La resistencia a compresión del concreto es uno de los parámetros más importantes en su control de calidad. Sin embargo, estos ensayos se realizan tras un periodo de curado que hace que los resultados disten de ser inmediatos a la fabricación del producto. Por lo tanto, se propone un modelo matemático fiable para obtener los resultados del ensayo en forma inmediata. Los modelos propuestos presentan coeficientes de correlación mayores a 0.9 y permiten reducir considerablemente el tiempo en obtener los resultados de la resistencia a compresión. de calidad.

\section{Use of Artificial Neural Networks for Modeling of the Test of Compressive strength of Construction Concrete According to the Standard ASTM C39/C 39 M}

\begin{abstract}
Two artificial neural network models for predicting the results of compressive strength test of a construction concrete after the curing period are proposed. The compressive strength of concrete is one of the most important variables in its quality control. However, these tests are carried out after a period of curing so results of the test are not immediately available. Therefore a reliable mathematical model that would obtain the test results immediately after the curing time These models present correlation coefficients higher than 0.9 and allow reducing the time to obtain the results of compressive strength tests.
\end{abstract}

Keywords: compressive strength, curing time, artificial neural networks, concrete, quality control. 


\section{INTRODUCCIÓN}

El concreto es, sin lugar a dudas, el material más utilizado en la construcción civil. Su fabricación se realiza a partir de la mezcla de componentes básicos: agua, cemento, agregados finos y gruesos. Las proporciones de dichos componentes, así como la inclusión de aditivos y otros factores, determinará la resistencia del concreto fabricado. Por otro lado, se sabe que la resistencia a compresión del hormigón evoluciona con el tiempo. Así se conoce que a los 7 se obtiene el $70-75 \%$ de la resistencia a 28 días y que a los 56 y 90 días se obtienen resistencias de un $10 \%$ a un $15 \%$ superiores a las obtenidas a los 28 días (Kosmatka et al., 2003). Para poder fijar un valor de referencia se toma universalmente como resistencia de referencia el valor obtenido mediante el ensayo a compresión a los 28 días (Kosmatka et al., 2003). Por lo tanto, los ensayos de compresión de bloques cilíndricos de concreto a 28 días es el utilizado en la práctica para el control de calidad del concreto. A partir de sus resultados se programar las operaciones de desencofrado, estimar la resistencia del concreto en estructuras, etc. Por lo tanto, es importante disponer de un modelo que permita predecir dicha resistencia a partir de los parámetros de fabricación a fin de evitar el tiempo de espera para el ensayo.

Existen numerosas investigaciones que relacionan y modelizan tanto la resistencia a compresión del concreto a partir de parámetros de su fabricación (Abrams, 1919; Yeh, 1998; Kosmatka et al. 2003; Ozturan et al., 2008; Caldarone, 2009) como la evolución de su endurecimiento. Sin embargo las correlaciones que se obtienen son generalmente bajas 0 , en el caso del endurecimiento, dichas relaciones se obtienen mediante ecuaciones diferenciales parciales no lineales (Martinelli et al, 2013), sólo resolubles de forma aproximada mediante el método de diferencias finitas.

Las redes neuronales artificiales son unas estructuras matemáticas que intentan imitar el funcionamiento de un cerebro biológico. Se componen de una serie de nodos o neuronas, interconectados entre sí, estructurados en una serie de capas. La capa de entrada recibe las señales del exterior y es la encargada de distribuir dichas señales hacia las capas internas (Fig. 1 y 2). Las capas internas son las que realizan los cálculos necesarios a fin de obtener una salida. Finalmente es la capa de salida la que muestra los resultados de la red. Estas estructuras son capaces de extraer conocimiento de una serie de datos de ejemplo, para luego aplicarlo a datos desconocidos. Este conocimiento se almacena en las conexiones entre las distintas neuronas que componen la red (Priore et al., 2002).

No existe una regla definida que nos indique cuántas neuronas debe tener la capa oculta y si ésta debe estar formada por una o más subcapas, sólo una serie de recomendaciones respecto al número de neuronas que debe haber en función de los datos disponibles (Sha y Edwards, 2007) o que la estructura de la red debe ser piramidal (Vanstone y Finnie, 2009), por lo que la única forma de obtener su estructura es mediante prueba y error (Lin y Tseng, 2000). Sí se debe tener en cuenta que una estructura con pocas neuronas puede no ser capaz de generalizar correctamente y que un número excesivo de neuronas no mejora sustancialmente la capacidad de la red dificultando su aprendizaje (Cheng, 1995). El carácter de aproximadores universales de las redes neuronales artificiales (Hornik, 1989; Hagan et al., 1996) las convierte en una herramienta muy útil de cara a la modelización de procesos en los que no interesan las relaciones entre las variables implicadas en él, sino la obtención de un resultado a partir de unos datos iniciales (De Veaux y Ungar, 1996). En los últimos años han tenido un gran desarrollo en el control de procesos industriales debido principalmente a su capacidad de modelar relaciones complejas que los sistemas tradicionales no han sido capaces de hacer y a predecir satisfactoriamente si las características de un producto se adecuan o no a las especificaciones correspondientes (Sukthomya y Tannock, 2005).

Estas estructuras han sido utilizadas en muy diversos ámbitos que van desde al control automático de condiciones ambientales (Henriquez y Palma, 2011) hasta análisis de imágenes de microscopio (Alves et al., 2011). Así mismo, han sido ampliamente utilizadas para la obtención de las propiedades mecánicas de distintos materiales como el cemento (Yeh, 1998; Baykasoğ et al., 2004; Cladera y Marí, 2004a; Cladera y Marí, 2004b; Ozturan et al., 2008; Prasad et al., 2009; Bilim et al., 2009; Sarıdemir, 2009; Özcan et al., 2009; Başyigit et al., 2010; Yaprak et al., 2013) en los que se busca predecir la resistencia a la compresión después del proceso de curado a partir de datos iniciales; algunos metales (Malinov et al., 2001; Hassan et al., 2009; Ozerdem y Kolukisa, 2009; Reddy et al., 2009) o el basalto (Çanakci y Pala, 2007). Para el tablero de partículas también se han utilizado para predecir su cohesión interna a partir de parámetros de fabricación (Cook y Whittaker, 1992; Cook y Chiu, 1997) o sus propiedades mecánicas de resistencia a la flexión, módulo de elasticidad y resistencia interna a partir de ensayos físicos (García Fernández et al., 2008) a fin de predecir posibles fallos en la producción sin tener que esperar a realizar dichos ensayos mecánicos. En este estudio se ha modelado, a partir de dos tipos de redes neuronales artificiales, el ensayo a compresión de bloques cilíndricos de concreto a partir de los parámetros de fabricación. Posteriormente se han comparado los resultados obtenidos por cada una de las redes a fin de utilizar en la modelización la más precisa. 


\section{MATERIALES Y MÉTODOS}

En este estudio se han utilizado 267 probetas de concreto fabricados con distintos tipos y cantidades de cemento, arena, piedra y agua. Las probetas para el ensayo de compresión se han fabricado de acuerdo a la norma ASTM C 192/C 192M (ASTM, 2000). Los ensayos de compresión se han realizado según la norma ASTM C39/C 39M (ASTM, 2001) tras diferentes periodos de curado. Los ensayos de compresión axial se realizaron en una máquina TONI-TECHNIK provista de una célula de $3.000 \mathrm{KN}$ y TINIUS OLSEN DE $1.500 \mathrm{KN}$. De todas las probetas se tomaron datos, que actuaron como variables de entrada, de cantidad de cemento, cantidad de agua, módulo de finura de la arena, módulo de finura de la piedra, tamaño nominal máximo de la piedra (TNM), peso específico de la arena, peso específico de la piedra, así como el tipo de cemento con el que está fabricado, periodo de curado y, como variable de salida, la resistencia a la compresión.

1) Cemento: Es el material que actúa como aglomerante de los otros materiales que componen el concreto. En este estudio se han utilizado cementos de tipo I a tipo V de acuerdo a la norma ASTM C150 (ASTM 2002).

2) Agua: El agua permite la hidratación del cemento además de brindar la trabajabilidad necesaria en la mezcla (slump o asentamiento). En este estudio se utilizó agua potable acorde a los requisitos de fabricación del concreto.

3) Módulo de finura: Es el coeficiente que permite detectar variaciones en la granulometría que podrían afectar el diseño de la mezcla.

4) TNM piedra: El tamaño nominal máximo se define como el diámetro correspondiente al tamiz anterior en el cual quedan retenidos entre el 5-15\% del material tamizado.

5) Arena: La cantidad de arena que se va a utilizar en la mezcla se define de acuerdo a las propiedades de ésta, tales como: granulometría, peso específico, contenido de material más fino que la malla 200 e impurezas orgánicas.

6) Piedra: Las piedras para uso en concreto se pueden ver afectadas por la forma (redondeada, angular), textura (lisa o rugosa). La cantidad de piedra dependerá del módulo de finura de la arena y el peso unitario del mismo.

7) Peso específico de los agregados: Se define como el peso por unidad de volumen. En el presente estudio se utilizaron agregados del país con valores dentro del rango de 2.500 a $2.800 \mathrm{~kg} / \mathrm{m} 3$.

8) Edad de ensayo: Es el parámetro crítico en la resistencia del concreto ya que ésta varía con el tiempo de curado. En el presente estudio se tomaron rangos de edad de ensayo entre 3 y 28 días.

A fin de obtener un proceso de curado óptimo, las probetas se sumergieron completamente en pozas de agua durante todo el proceso.

Para la modelización del ensayo a compresión del concreto se han utilizado dos tipos de redes neuronales artificiales, una red neuronal tipo perceptrón multicapa y una red Elman, ambas con funciones de transferencia tipo tangente hiperbólica sigmoidea (Fig. 1 y 2).

Como primer paso al diseño de la red se realizó un análisis de componentes principales del vector de entrada, con el fin de determinar si existe algún tipo de correlación entre las variables de entrada, mejorando los resultados de la red (Demuth et al, 2002; Clark et al, 2012). Este proceso se realiza automáticamente, por un programa desarrollado ad hoc (Fig. 1), antes de introducir los datos en la red. Como criterio para la selección de las variables se tomo que su contribución a la variabilidad de la muestra fuera superior al 2\% (Sarle 1997, Demuth et al. 2002).El programa elimina automáticamente las variables con una contribución inferior al $2 \%$.

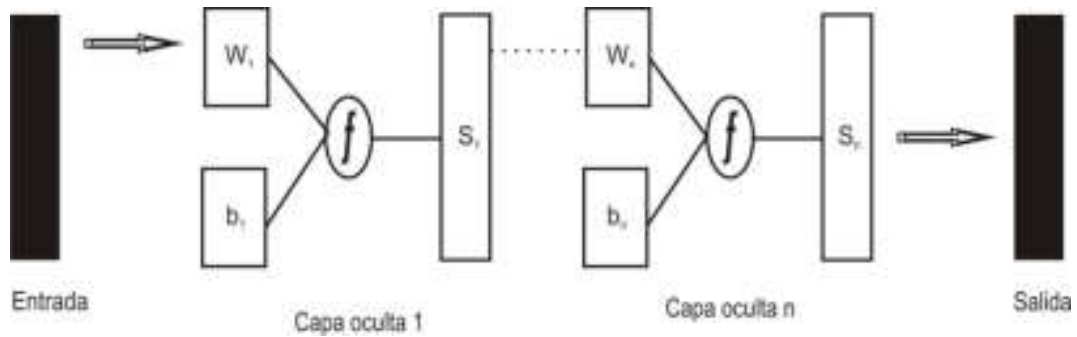

Fig. 1: Esquema de un perceptrón multicapa. $W_{i}$ : Neuronas de la i-ésima capa interna. bi: Sesgo de la iésima capa interna. $S_{\mathrm{i}}$ : salida de la i-ésima capa interna. 


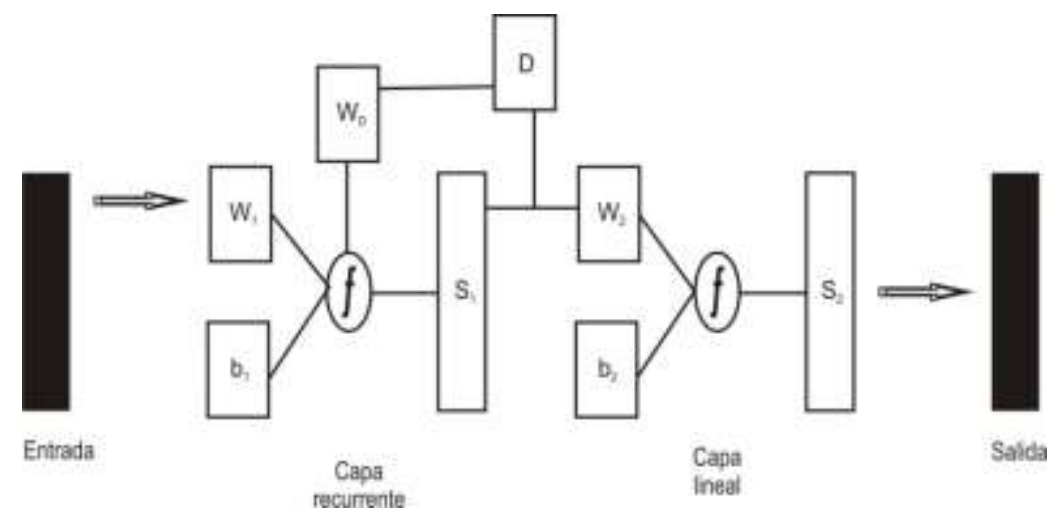

Fig. 2: Esquema de una red de Elman. $W_{i}$ : Neuronas de la i-ésima capa interna. $b_{i}$ : Sesgo de la i-ésima capa interna. Si: salida de la i-ésima capa interna. D: Capa neuronal recurrente.

El perceptrón multicapa es un tipo de red neuronal con conexiones hacia adelante mientras que la red de Elman es un tipo de red neuronal recurrente, basada en el perceptrón multicapa, muy utilizada en la modelización de series temporales. Sin embargo, pese a que su recurrencia le permite modelizar patrones temporales, su facilidad de desarrollo ha extendido su uso también a la modelización de patrones estáticos. Por otra parte, el carácter de aproximador universal de funciones del perceptrón multicapa (Hornik, 1989; Hagan et al., 1996) hace que las redes seleccionadas sean muy adecuadas para modelizar los resultados del ensayo de compresión de concreto.

Como función de transferencia se ha optado por la función tangente hiperbólica sigmoidea (Ecuación. 1), la cual es equivalente a la tangente hiperbólica, solo que mejora el rendimiento de la red al producir una salida más rápida (Demuth et al., 2002).

$$
f(\theta)=\frac{2}{1+e^{(-2 \theta)}}-1
$$

Dónde $f(\theta)$ es el valor de salida de la neurona y $\theta$ el valor de entrada de la neurona.

A fin de mejorar el rendimiento de la red y debido a que los valores de salida de la función de transferencia están en el intervalo $(-1,1)$ (Rafiq et al., 2001) se transformaron los datos mediante la función mapstd de MATLAB, en forma afín y biunívoca, al intervalo $[0,1]$ y en seguida con processpca se realizó un análisis de componentes principales eliminando las variables redundantes dentro del conjunto de entrada. El método de entrenamiento de la red se ha realizado mediante aprendizaje supervisado basado en técnicas de minimización local de funciones de varias variables (Hagan et al., 1996; Isasi y Galván, 2004). Para el entrenamiento se ha utilizado el algoritmo de retropropagación resilente, el cual mejora los resultados del aprendizaje para el caso de funciones de transferencia sigmoidea (Demuth et al., 2002).

Para evitar el problema de sobreentrenamiento, que deteriora la capacidad de generalización de la red, se ha optado por el método de early-stopping. El sobreentrenamiento de una red neuronal se manifiesta por un descenso del error en el conjunto de entrenamiento frente a un aumento en el conjunto de validación, es síntoma de que la red se está adaptando perfectamente a los datos del conjunto de entrenamiento pero ha perdido su capacidad de generalización (Hagan et al., 1996). Para evitarlo se ha comparado cada 1000 ciclos de entrenamiento el error en ambos conjuntos, dando por finalizado el proceso en el momento en que el error del conjunto de validación aumenta frente a un descenso en el conjunto de entrenamiento. A tal fin, El conjunto inicial de datos ha sido dividido en tres grupos elegidos aleatoriamente y sin repetición; el de entrenamiento ( 164 datos, $61.4 \%$ del total), el de validación ( 46 datos, $17.2 \%$ del total) y el de comprobación (57 datos, $21.3 \%$ del total) porcentaje muy similar al utilizado por García Fernández et al., (2012) en su estudio. Los dos primeros conjuntos se utilizarán en el proceso de desarrollo de la red. El tercer conjunto, el de comprobación, se utilizará para evaluar el grado de generalización de la red evitando así cualquier tipo de sesgo introducido por los conjuntos de entrenamiento y validación (Bishop 1995; Hagan et al., 1996).

Se ha optado por diseñar un programa automático de separación de conjuntos (Fig. 3) y no utilizar el método implementado automáticamente en MATLAB, el cuál emplea clases de equivalencia de enteros módulo 4, a fin de garantizar la no existencia de diferencias significativas entre los conjuntos. 


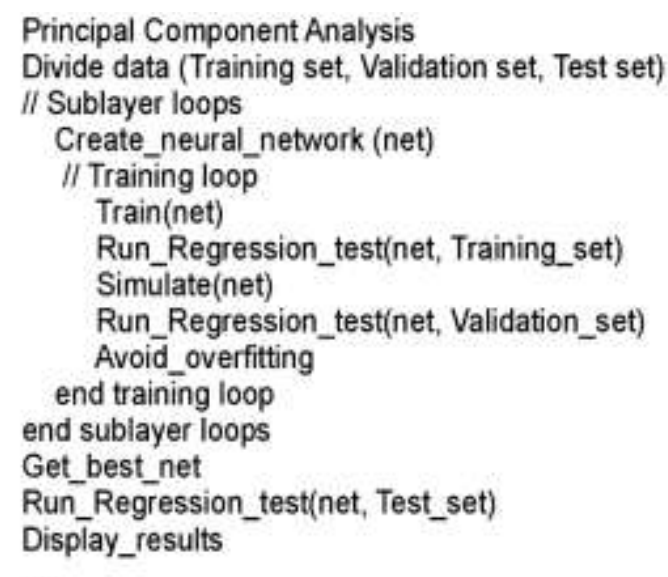

Fig. 3: Pseudocódigo del programa desarrollado.

Para evaluar el resultado final se va a utilizar el coeficiente de regresión $(R)$, el coeficiente de determinación $\left(R^{2}\right)$, la raíz del error cuadrático medio (RMSE) y el cociente $\left(V_{\text {test }} / V_{\text {red }}\right)$ entre los entre los resultados de la red y los reales y el tiempo empleado en su desarrollo. Para los desarrollos de las redes neuronales se ha desarrollado un programa específico en lenguaje MATLAB ${ }^{\circledR}$, utilizando el Neural Network Toolbox ${ }^{\circledR}$ ver. 4.0.2, perteneciente al programa MATLAB ${ }^{\circledR}$ Ver. 6.5.0. Release 13.

\section{RESULTADOS Y DISCUSIÓN}

Los resultados obtenidos en los ensayos realizados se recogen en la siguiente tabla (Tabla 1). El análisis de componentes principales arroja un nivel de contribución a la variabilidad de la muestras superior al $2 \%$ para cada uno de los componentes. Las redes óptimas obtenidas son un perceptrón multicapa con dos capas ocultas de 10 y 4 neuronas ocultas y una red Elman de tres capas ocultas de 9, 8 y 3 neuronas cada una. Los resultados de los procesos de entrenamiento, validación y comprobación se recogen en la siguiente tabla (Tabla 2). Las figuras 4 y 5 muestran las correlaciones en los conjuntos de comprobación para la red tipo perceptrón y tipo Elman.

Tabla 1: Resultados de los ensayos. Mfa: módulo de finura de arena; MFp: módulo de finura de piedra; TNM: Tamaño nominal máximo de piedra; PEa: peso específico arena; PEp: Peso específico piedra; Eens: Edad del ensayo; R: Resistencia a compresión.

\begin{tabular}{|c|c|c|c|c|c|c|}
\hline & \multicolumn{6}{|c|}{ Propiedad } \\
\hline & $\begin{array}{c}\text { Cemento } \\
(\mathrm{kg} / \mathrm{m} 3)\end{array}$ & $\begin{array}{c}\text { Agua } \\
\text { (L/m3) }\end{array}$ & \multicolumn{2}{|c|}{ Mfa } & Mfp & TNM \\
\hline Media & 364.47 & 214.32 & \multicolumn{2}{|c|}{3.04} & 7.27 & 0.98 \\
\hline Desv. típica & 60.19 & 7.51 & \multicolumn{2}{|c|}{0.46} & 0.44 & 0.14 \\
\hline Mínimo & 238.00 & 190.00 & \multicolumn{2}{|c|}{1.89} & 5.37 & 0.50 \\
\hline \multirow[t]{3}{*}{ Máximo } & 583.00 & 232.00 & & & 7.96 & 1.50 \\
\hline & \multicolumn{6}{|c|}{ Propiedad } \\
\hline & $\begin{array}{c}\text { Arena } \\
(\mathrm{kg} / \mathrm{m} 3)\end{array}$ & $\begin{array}{c}\text { Piedra } \\
\text { (kg/m3) }\end{array}$ & $\begin{array}{c}\text { PEa } \\
(\text { Ton/m3) }\end{array}$ & $\begin{array}{c}\text { PEp } \\
\text { (Ton/m3) }\end{array}$ & $\begin{array}{l}\text { Eens } \\
\text { (días) }\end{array}$ & $\begin{array}{c}\mathrm{R} \\
(\mathrm{kg} / \mathrm{cm} 2)\end{array}$ \\
\hline Media & 805.85 & 924.05 & 2.62 & 2.81 & - & 224.10 \\
\hline Desv. típica & 91.96 & 96.77 & 0.07 & 0.68 & - & 64.10 \\
\hline Mínimo & 377.00 & 200.00 & 2.28 & 2.46 & 3.00 & 65.54 \\
\hline Máximo & 1005.00 & 1319.00 & 2.72 & 7.46 & 28.00 & 369.06 \\
\hline
\end{tabular}

Tabla 2: Resultados del proceso de desarrollo de la red.

\begin{tabular}{|c|c|c|c|c|c|c|c|}
\hline Red & Proceso & $\mathrm{R}$ & $\mathrm{R}^{2}$ & Ec. recta & RMSE & $\mathrm{V}_{\text {test }} / \mathrm{V}_{\text {red }}$ & $\begin{array}{c}\text { Tiempo } \\
\text { desarrollo (min) }\end{array}$ \\
\hline \multirow{3}{*}{$\begin{array}{l}\text { Perceptrón } \\
{\left[\begin{array}{lllll}11 & 10 & 4 & 1\end{array}\right]}\end{array}$} & Entrenamiento & 0.975 & 0.951 & $y=0.93 \cdot x+16$ & 13.92 & 1.02 & \multirow{3}{*}{19.23} \\
\hline & Validación & 0.929 & 0.863 & $y=0.92 \cdot x+18$ & 24.54 & 1.00 & \\
\hline & Comprobación & 0.933 & 0.870 & $y=0.85 \cdot x+29$ & 23.49 & 1.01 & \\
\hline \multirow{3}{*}{$\begin{array}{c}\text { Elman } \\
{\left[\begin{array}{lllll}11 & 9 & 8 & 3 & 1\end{array}\right]}\end{array}$} & Entrenamiento & 0.985 & 0.971 & $y=0.97 \cdot x+7.8$ & 11.07 & 1.01 & \multirow{3}{*}{28.18} \\
\hline & Validación & 0.934 & 0.871 & $y=0.89 \cdot x+20$ & 22.80 & 0.99 & \\
\hline & Comprobación & 0.939 & 0.882 & $y=0.86 \cdot x+31$ & 22.32 & 1.02 & \\
\hline
\end{tabular}




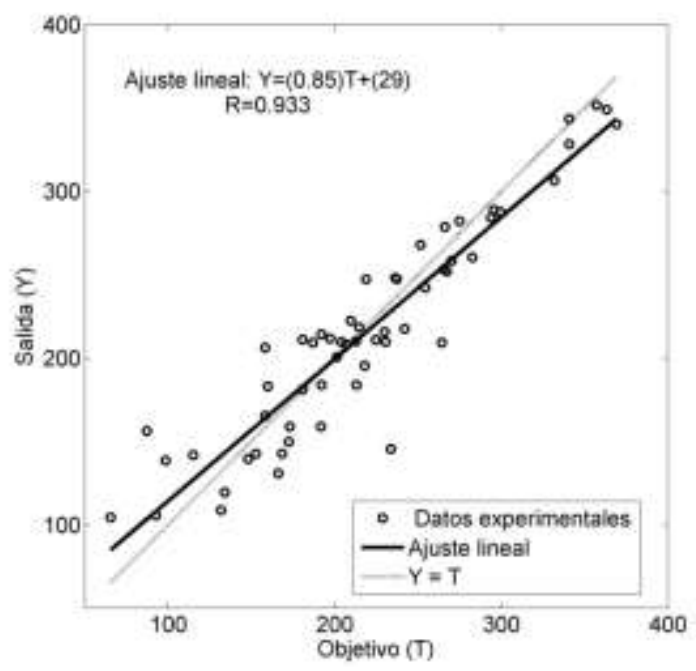

Fig. 4: Correlación en el conjunto de comprobación para la red tipo perceptrón

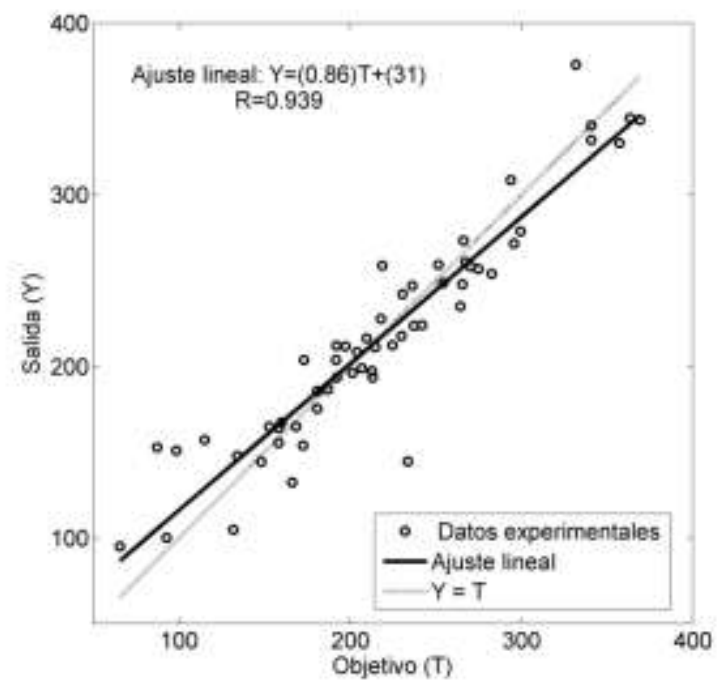

Fig. 5: Correlación en el conjunto de comprobación para la red tipo Elman

Los coeficientes de determinación obtenidos en el conjunto de comprobación indican que los modelos desarrollados consiguen explicar al menos el $87 \%$ de la variabilidad de las muestras. Los resultados obtenidos durante el proceso de desarrollo de la red, con coeficientes de correlación comprendidos entre 0.93 y 0.98 , son coherentes con los obtenidos por otros autores consultados, los cuales obtuvieron coeficientes de correlación entre 0.81 y 0.98 (Lee, 2003; Oztas et al., 2006; Ukrainczyk y Ukrainczyk, 2008; Yapak et al., 2013). Igualmente los coeficientes de determinación obtenidos $\left(R^{2}=0.87\right.$ y $\left.R^{2}=0.88\right)$ son superiores a los obtenidos por Yeh (1998) y similares a los obtenidos por Ozturan et al. (2008) y Prasad et al. (2009) los cuales obtuvieron coeficientes máximos entre 0.78 y 0.92 .

Yeh (1998) y Ozturan et al. (2008) también modelizaron la resistencia del concreto mediante modelos de regresión. Sin embargo obtuvieron resultados inferiores a los obtenidos en este estudio. Así, para dichos modelos, Yeh (1998) obtuvo coeficientes de determinación entre 0.46 y 0.89 y Ozturan et al. (2008) obtuvieron coeficientes de determinación entre 0.55 y 0.79 .

Por otro lado, estos resultados se encuentran dentro de los intervalos que otros autores han obtenido al modelizar otros materiales Malinov et al., (2001) obtuvieron correlaciones entre 0.60 y 0.97 en su modelización de las propiedades mecánicas de las aleaciones de titanio. Mc Bride et al., (2004), obtuvieron coeficientes de correlación entre 0.94 y 0.97 en la modelización de las propiedades mecánicas de aleaciones de aluminio.

Finalmente los coeficientes $\mathrm{V}_{\text {test }} / \mathrm{V}_{\text {red }}$ calculados se encuentran dentro de los intervalos obtenidos por Cladera y Marí (2004a, 2004b), los cuales obtuvieron coeficientes entre 0.99 y 1.02 en sus estudios de la modelización del ensayo a cortante del concreto de alta resistencia. 
Por lo tanto, se puede comprobar que los resultados obtenidos están dentro de los márgenes considerados por otros autores como aceptables en sus trabajos de investigación y, consecuentemente, podemos considerar ambas redes desarrolladas como aptas para la modelización del ensayo de compresión del concreto.

Los resultados obtenidos por la red de tipo perceptrón y la red de tipo Elman son muy similares. Sin embargo el perceptrón, el cual tiene dos capas ocultas de 10 y 4 neuronas, presenta una menor complejidad interna, frente a la red de Elman que tiene tres capas ocultas de 9, 8 y 3 neuronas cada una. Esta circunstancia, unida a que su tiempo de desarrollo es sensiblemente inferior, hace que sea el perceptrón el más adecuado para resolver de forma efectiva el problema de la modelización del ensayo de compresión del concreto.

\section{CONCLUSIONES}

Se ha obtenido la modelización mediante redes neuronales artificiales del ensayo de resistencia a compresión a partir de los parámetros de fabricación con un coeficiente de correlación superior a 0.93.

Se ha introducido una nueva técnica de modelización para resolver los problemas de obtención de la resistencia a compresión del concreto tras un periodo de curado, reduciendo considerablemente el tiempo de obtención de los resultados.

Los resultados obtenidos reflejan una gran similitud entre la red tipo perceptrón y la red tipo Elman. Sin embargo la mayor simplicidad de la red tipo perceptrón y su menor tiempo de desarrollo la hace más adecuada para su uso en este problema concreto.

\section{AGRADECIMIENTOS}

Este trabajo se ha desarrollado con el soporte del Instituto General de Investigación de la Universidad nacional de Ingeniería (Concurso de Proyectos de Investigación Interdisciplinario 2012 - II). El Grupo de Investigación quiere agradecer al Dr. Perdo Espinoza por su ayuda en el desarrollo de las redes neuronales artificiales y al Ayudante-Alumno, Anthony Pajuelo del Laboratorio de Ensayo de Materiales de la Facultad de Ingeniería Civil perteneciente a la Universidad Nacional de Ingeniería (LEM-FIC-UNI) por su contribución en la realización de los ensayos y el mantenimiento de la base de datos.

\section{REFERENCIAS}

Alves, M.L., B.B. Ferreira y F.R. Leta, Evaluación de parámetros de rugosidad usando análisis de imágenes de diferentes microscopios ópticos y electrónicos, Información Tecnológica: 22(4), 129-146 (2011).

Abrams, D.A., Design of concrete mixtures, Structural Materials Research Laboratory, Lewis Institute. Chicago, USA (1919).

ASTM C 39/C 39M: The American Society for Testing Materials, Standard Test Method for Compressive Strength of Cylindrical Concrete Specimens, West Conshohocken (PA), USA (2001).

ASTM C 150: The American Society for Testing Materials Standard Specification for Portland Cement, West Conshohocken (PA), USA (2002).

ASTM C 192/C 192M: The American Society for Testing Materials Standard Practice for Making and Curing Concrete Test Specimens in the Laboratory, West Conshohocken (PA), USA (2000).

Başyigit, C., I. Akkurt, S. Kilincarsian y A. Beycioglu, Prediction of compressive strength Of heavyweight concrete by ANN and FL models, Neural Computing and Applications: 19, 507-513 (2010).

Baykasoğ, A., T. Delhi y S. Tanış, Prediction of cement strength using soft computing techniques, Cement and Concrete Research: 34, 2083-2090 (2004).

Bishop, C.M., Neural networks for pattern recognition, Oxford University Press, Oxford, Gran Bretaña (1995).

Bilim, C., C.D. Atiş, H. Tanyildizi, y O. Karahan, Predicting the compressive strength of ground granulated blast furnace slag concrete using artificial neural networks, Advances in Engineering Software: 40, 334-340 (2009). 
Caldarone, M.A., High-Strength Concrete: A Practical Guide, Taylor and Francis, New York, USA (2009).

Cladera, A. y A.R. Marí, Shear design for reinforced normal and high-strength concrete beams using artificial neural networks. Part I: beams without stirrups, Engineering structures: 26 (7), 917-926 (2004)

Cladera, A. y A.R. Marí, Shear design procedure for reinforced normal and high-strength concrete beams using artificial neural networks. Part II: Beams with stirrups, Engineering structures: 26 (7), 927-936 (2004)

Çanakci, H. y M. Pala, Tensile strength of basalt from a neural network, Engineering Geology: 94, 10-18 (2007).

Cheng C.S., A multi-layer neural network model for detecting changes in the process mean, Computers and Industrial Engineering: 28, 51-61 (1995).

Cook, D.F. y C.C. Chiu, Predicting the internal bond strength of particleboard, utilizing a radial basis function neural network, Engineering Applications of Artificial Intelligence: 10(2), 171-177 (1997).

Cook, D.F. y A.D. Whittaker, Neural network models for prediction of process parameters in wood products manufacturing, Proceedings of 1st Industrial Engineering Research Conference, Chicago, USA: 209-211 (1992).

Demuth, H., M. Beale y M. Hagan, Neural Network Toolbox User's Guide, Version 4, The MathWorks Inc., Natick, USA (2002).

De Veaux R.D. y L.G. Ungar, A brief introduction to neural networks, (1996).

http://www.cis.upenn.edu/ ungar/Datamining/Publications/nnet-intro.pdf. Acceso: 25 de febrero (2013).

García Fernández, F., L.G. Esteban, P. de Palacios, N. Navarro y M. Conde, Prediction of standard particleboard mechanical properties utilizing an artificial neural network and subsequent comparison with a multivariate regression model, Investigación Agraria: Sistemas y Recursos Forestales: 17(2), 178-187 (2008).

García Fernández, F., P. de Palacios, L. García Esteban, A. García-Iruela, B. González Rodrigo y E. Menasalvas, Prediction of MOR and MOE of structural plywood board using an artificial neural network and comparison with a multivariate regression model, Composites: Part B: 43, 3528-3533 (2012).

Hagan, M.T., H.B. Demuth y M. Beale, Neural Network Design, PWS Pub. Co, Boston, USA (1996).

Hassan, A.M., A. Alrashdan, M.T. Hayajneh y A.T. Mayyas, Prediction of density, porosity and hardness in aluminium-cooper-based composite materials using artificial neural network, Journal of Materials Processing Technology: 209, 894-899 (2009).

Henriquez, M.R. y P.A. Palma, Control automático de condiciones ambientales en domótica usando redes neuronales, Información Tecnológica: 22(3), 125-139 (2011).

Hornik, K., Multilayer Feedforward Networks are Universal Approximators, Neural Networks: 2, 359-366 (1989).

Isasi P, y I.M. Galván, Redes Neuronales Artificiales, un enfoque práctico, Pearson Educación S.A, Madrid, España (2004).

Kosmatka, S.H., B. Kerkhoff y W.C. Panarese, Design and Control of Concrete Mixtures, Portland Cement Association. Skokle, Illinois, USA (2003).

Lee, S.C., Prediction of concrete strength using artificial neural networks, Journal of Engineering Structure: 25, 849-857 (2003).

Lin T.Y. y C.H. Tseng, Optimum design for artificial networks: an example in a bicycle derailleur system, Engineering Application of Artificial Intelligence: 13, 3-14 (2000).

Martinelli E., A.B. Koenders y A.Caggiano, A numerical recipe for modelling hydration and heat flow in hardening Concrete, Cement \& Concrete Composites: 40, 48-58 (2013). 
Malinov, S., W. Sha y J.J. McKeown, Modelling the correlation between processing parameters and properties in titanium alloys using artificial neural networks, Computational Materials Science: 21, 375-394 (2001).

McBride, J., S. Malinov, y W. Sha, Modelling tensile properties of gamma-based titanium aluminides using artificial neural network, Material Science and Engineering A: 384, 129-135 (2004).

Özcan, F., C.D. Atiş, O. Karahan, E. Uncuoğlu y H. Tanyildizi, Comparison of an artificial neural network and fuzzy logic models for prediction of long-term compressive strength of silica fume concrete, Advances in Engineering Software: 40, 856-863 (2009).

Oztas, A., M. Pala, E. Ozbay, E. Kanka, A. Caglar y M.A. Bhatti, Predicting the compressive strength and slum of high strength concrete using neural network, Construction and Building Materials: 20-9, 769-775 (2006).

Ozturan, M., B. Kutlu, y T. Ozturan, Comparison of concrete strength prediction techniques with artificial neural network approach, Building Research Journal: 56, 23-36 (2008).

Ozerdem, M.S. y S. Kolukisa, Artificial neural network approach to predict the mechanical properties of CuSn-Pb-Zn-Ni cast alloys, Materials and Design: 30, 764-769 (2009).

Prasad, B.K.R., H. Eskandari y B.V.V. Reddy, Prediction of compressive strength of SCC and HPC with hay volumen fly ash using ANN, Construction and Building Materials: 23, 117-128 (2009).

Priore, P., D. de La Fuente, R. Pino y J. Puente, Utilización de las redes neuronales en la toma de decisiones. Aplicación a un problema de secuenciación, Anales de Mecánica y Electricidad: 79(6), 28-34. (2002).

Rafiq, M.Y., G. Bugmann y D.J. Easterbrook, Neural Network design for engineering applications, Computers \& Structures: 79, 1541-1552 (2001).

Reddy, N.S., J. Krishnaiah, S.G. Hong y J.S. Lee, Modeling medium carbon steels by using artificial neural networks, Materials Science and Engineering A: 508, 93-105 (2009).

Sarle, W.S., Neural Network FAQ, part 1 of 7: Introduction, periodic posting to the Usenet newsgroup comp.ai.neural-nets (1997) URL: ftp://ftp.sas.com/pub/neural/FAQ.html Acceso: 20 de enero (2014)

Sarıdemir, M., Prediction of compressive strength of concretes containing metakaolin and silica fume with neural networks, Advances in Engineering Software: 40, 350-355 (2009).

Sha, W. y K.L. Edwards, The use of artificial neural networks in materials science based research, Materials and Design: 6, 1747-1752 (2007).

Sukthomya, W. y J. Tannock, The training of neural networks to model manufacturing processes, Journal of Intelligent Manufacturing: 16, 39-51 (2005).

Ukrainczyk, N. y V. Ukrainczyk; A neural network method for analysing concrete durability, Magazine of Concrete Research: 60(7), 475-486 (2008).

Vanstone, B. y G. Finnie, An empirical methodology for developing stockmarket Trading Systems using artificial neural Networks, Expert systems with Applications: 36, 6668-6680 (2009).

Yaprak, H., A. Karaci, y I. Demir, Prediction of the effect of varying cure conditions and w/c ratio on the compressive strength of concrete using artificial neural networks, Neural Computing and Applications: 22 , 133-141 (2013).

Yeh, I.C., Modeling of strength of high-performance concrete using artificial neural networks, Cement and Concrete Research: 28(12), 1797-1808 (1998). 
